\title{
A!
}

This is an electronic reprint of the original article.

This reprint may differ from the original in pagination and typographic detail.

Niskanen, Antti O.; Kivioja, Jani; Seppä, Heikki; Pekola, Jukka

\section{Evidence of Cooper-pair pumping with combined flux and voltage control}

Published in:

Physical Review B

DOI:

10.1103/PhysRevB.71.012513

Published: 26/01/2005

Document Version

Publisher's PDF, also known as Version of record

Please cite the original version:

Niskanen, A. O., Kivioja, J., Seppä, H., \& Pekola, J. (2005). Evidence of Cooper-pair pumping with combined flux and voltage control. Physical Review B, 71(1), 1-4. [012513]. https://doi.org/10.1103/PhysRevB.71.012513

This material is protected by copyright and other intellectual property rights, and duplication or sale of all or part of any of the repository collections is not permitted, except that material may be duplicated by you for your research use or educational purposes in electronic or print form. You must obtain permission for any other use. Electronic or print copies may not be offered, whether for sale or otherwise to anyone who is not an authorised user. 


\title{
Evidence of Cooper-pair pumping with combined flux and voltage control
}

\author{
Antti O. Niskanen, ${ }^{1,2, *}$ Jani M. Kivioja, ${ }^{2}$ Heikki Seppä, ${ }^{1}$ and Jukka P. Pekola ${ }^{2}$ \\ ${ }^{1}$ VTT Information Technology, Microsensing, P. O. Box 1207, FIN-02044 VTT, Finland \\ ${ }^{2}$ Low Temperature Laboratory, Helsinki University of Technology, P. O. Box 2200, FIN-02015 HUT, Finland
}

(Received 24 September 2004; published 26 January 2005)

\begin{abstract}
We have experimentally demonstrated pumping of Cooper pairs in a single-island mesoscopic structure. The island was connected to leads through SQUID (superconducting quantum interference device) loops. Synchronized flux and voltage signals were applied whereby the Josephson energies of the SQUIDs and the gate charge were tuned adiabatically. From the current-voltage characteristics one can see that the pumped current increases in $1 e$ steps which is due to quasiparticle poisoning on the measurement time scale, but we argue that the transport of charge is due to Cooper pairs.
\end{abstract}

DOI: 10.1103/PhysRevB.71.012513

PACS number(s): 74.78.Na, 74.50.+r, 73.23.-b

A device that yields a dc current in response to an ac signal at frequency $f$ according to the relation $I=Q f$ is called a charge pump. In the case of electron pumps $Q=m e$ while for Cooper pair pumps $Q=2 m e$, where $m$ is an integer denoting the number of charges being pumped per cycle. Typically pumping electrons in mesoscopic structures requires an array of at least three tunnel junctions with voltage gates coupled to the islands in between the junctions. A Cooper pair pump is obtained when the tunnel junctions are replaced by Josephson junctions. These devices appear at first sight to be very similar and actually the very same samples may serve as both Cooper pair and electron pumps depending on whether the device is in the superconducting state or not. However, major differences exist. Besides the doubled charge in the superconducting state, the nature of the tunneling processes is very different, too. Electrons can tunnel downhill in energy due to the inherent dissipation mechanisms in normal metals with the relevant time scale given by the $R C$ time constant, where $R$ is the tunnel resistance and $C$ is the tunnel capacitance. Cooper pairs, on the other hand, try to conserve their energy, and in the absence of an electromagnetic environment, (i.e., zero impedance), only elastic processes are possible. Their maximum pumping frequency is proportional to $E_{\mathrm{J}}^{2} /\left(E_{\mathrm{C}} \hbar\right)$, where $E_{\mathrm{J}}$ and $E_{\mathrm{C}}$ are the Josephson and charging energies, respectively. What is more, superconducting circuits may behave coherently in the quantummechanical sense. The first attempt to pump Cooper pairs dates back to over a decade ago. ${ }^{1}$ However, Cooper pair pumps have not been even nearly as accurate as singleelectron pumps. The best example of the latter ones is the NIST seven-junction pump. ${ }^{2}$ The motivation behind pumping Cooper pairs is twofold. First of all, Cooper pair pumps are hoped to be able to pump larger currents than their normal state counterparts while still being accurate. This is roughly because increasing $E_{\mathrm{J}}^{2} /\left(E_{\mathrm{C}} \hbar\right)$ is easier than increasing $1 /(R C)$. Secondly, the operation of Cooper pair pumps is interesting from the point of view of secondary "macroscopic" quantum phenomena and the structures are quite similar to the superconducting qubits (see, e.g., Refs. 3 and 4). Pumping of electrons using surface acoustic waves is another active field of study (see, e.g., Ref. 5).

In this work we report on the experimental demonstration of pumping Cooper pairs in a structure nicknamed the Cooper pair "sluice" introduced and theoretically analyzed recently by us (see Ref. 6). The device is particularly simple; it has just one superconducting island, like the single Cooper pair transistor, but the bare Josephson junctions are replaced by superconducting quantum interference device (SQUID) loops. The device may be alternatively viewed as a tunable Cooper pair box, a Josephson charge qubit. ${ }^{7}$ Here the control is achieved via adiabatically manipulating both the fluxes through the two loops and the gate voltage. Ideally the SQUIDs act as tunable Josephson junctions whose coupling energy can be varied between a value close to zero and the sum of the couplings of the individual junctions. First we describe the experimental setup and discuss the theoretical idea briefly. Then we present measured data of the pumping experiment. We demonstrate that the pumped current obeys nicely the theoretical predictions. We also comment on possible ways of improving the results should the device be used in applications and discuss the significance of the results.

Figure 1 shows a scanning electron micrograph (SEM) image of the sample used in the experiments along with a schematic of the measurement setup in Fig 1(c). The device was fabricated out of aluminum using standard e-beam lithography and two-angle shadow evaporation. It consists of a superconducting island that connects to the leads via SQUID loops. These are relatively large $(10 \mu \mathrm{m}$ by $100 \mu \mathrm{m})$ in order to have good inductive coupling but the island and the junctions are still small such that the charging energy is large enough $(\approx 1 \mathrm{~K})$ to suppress thermal effects. The sample was attached to a dilution cryostat with a base temperature of $20 \mathrm{mK}$ with the rf lines connected.

Ideally, the pumping of $m$ Cooper pairs is achieved by applying the three pulses in Fig. 2(b) through the attenuated rf lines. The upmost signal is applied to the gate while the two lower ones represent the currents flowing in the input coils. Two different versions of the gate pulse are shown, one for pumping "forward" and one for pumping "backward." To understand how the device works, it is instructive to look at the Hamiltonian of the device, which reads

$$
\begin{aligned}
\hat{H}= & E_{\mathrm{C}}\left(\hat{n}-n_{\mathrm{g}}\right)^{2}-E_{\mathrm{J}}^{1}\left(\Phi_{1}\right) \cos (\phi+\varphi / 2) \\
& -E_{\mathrm{J}}^{2}\left(\Phi_{2}\right) \cos (\varphi / 2-\phi) .
\end{aligned}
$$

Here $E_{\mathrm{C}}=2 e^{2} / C_{\Sigma}$ is the charging energy for Cooper pairs 


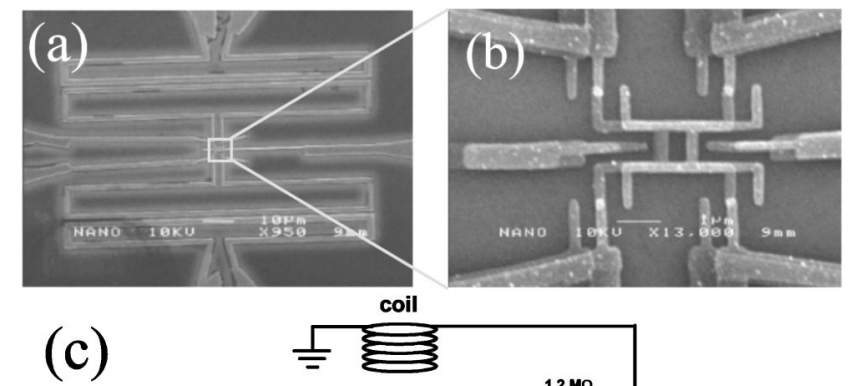

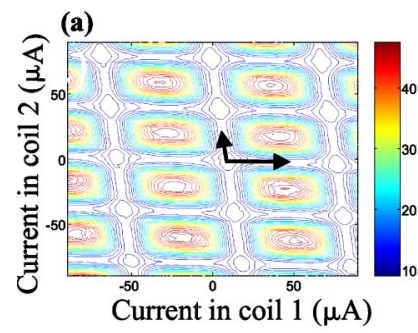

(b)

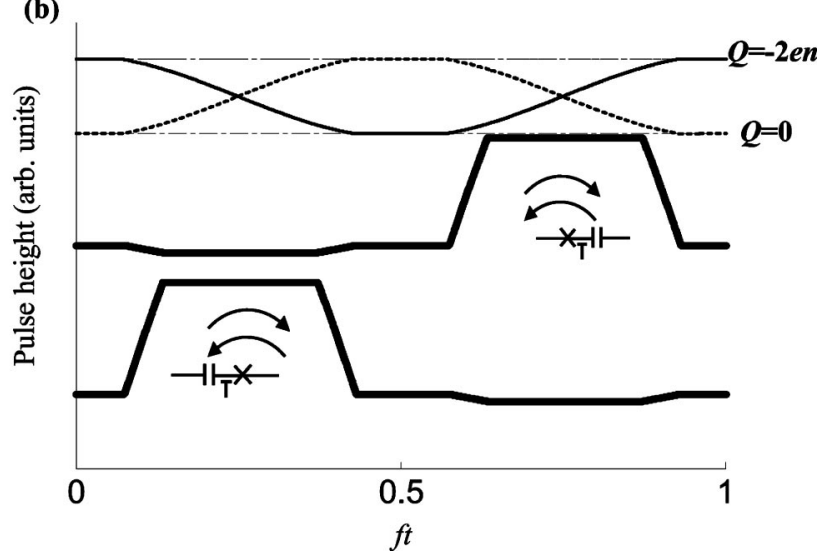

FIG. 1. (a) Scanning electron micrograph of the sample. The two input coils can be seen on the top and bottom, respectively. The gate extends to the far right and the gate capacitance is $C_{\mathrm{g}}$ $=0.24 \mathrm{fF}$ based on dc measurements. The current flows between the two leads on the left side. (b) Closeup of the island. The measured total capacitance of the island is $3.7 \mathrm{fF}$ which corresponds to a charging energy of about $1 \mathrm{~K}$ for Cooper pairs. The maximum $E_{\mathrm{J}}$ per SQUID is estimated to be around $0.5 \mathrm{~K}$ based on the normal state resistance. (c) Schematic illustration of the measurement setup. We used commercial room temperature electronics for the current measurement and three synchronized arbitrary waveform generators for the control pulse. The external coil for tuning the background of the SQUIDs is at $20 \mathrm{mK}$. The voltage biasing happens via voltage division through resistive lines. A surface mount capacitor of $680 \mathrm{pF}$ and an on-chip capacitor on the order of $10 \mathrm{pF}$ (indicated by $C_{\mathrm{s}}$ ) were also used.

where $C_{\Sigma}$ is the total capacitance seen from the island. Furthermore, $E_{\mathrm{J}}^{j}$ with $j=1,2$ are the (signed) Josephson energies of the two SQUIDs which can be tuned with the external fluxes $\Phi_{j}$. For identical junctions $E_{\mathrm{J}}^{j}=E_{\mathrm{J}}^{\max } \cos \left(\pi \Phi_{j} / \Phi_{0}\right)$, where $\Phi_{0} \approx 2 \times 10^{-15} \mathrm{~Wb}$ is the flux quantum and $E_{\mathrm{J}}^{\max }$ is proportional to the critical current $I_{\mathrm{C}}$ of the individual junctions via $E_{\mathrm{J}}^{\max }=(\hbar / e) I_{\mathrm{C}}$. Furthermore, $n_{\mathrm{g}}=C_{\mathrm{g}} V_{\mathrm{g}} / 2 e$ is the gate charge in $2 e$ units, $\hat{n}$ is the number operator for Cooper pairs, $\phi$ is the phase on the island, and their commutator is $[\hat{n}, \phi]=i$. The environment couples to the pump through $\varphi$ which is the phase difference over the pump. If the SQUIDs were to have perfectly identical junctions as well as vanishing self-inductance and if the flux control were perfect, then the effective Josephson couplings could be set to zero.

Figure 2(a) shows a contour plot based on the measurement of the current through the device at a constant voltage against the dc currents in the two input coils. Along the lines of minimum current the flux through either of the loops is $(k+1 / 2) \Phi_{0}$, where $k$ is an integer. The measurement reveals
FIG. 2. (a) Contour plot of the measured dc current at constant voltage against dc currents in the two input coils. The total variation in the current is around $40 \mathrm{pA}$ at this bias point $(150 \mu \mathrm{V})$. The arrow line indicates the path along which the flux pulsing is performed in the pumping experiment. The lines of minimum current along which the arrows are aligned are the lines along which half a flux quantum threads one of the two SQUIDs. The slight tilting of the lines is a signature of the inductive cross-coupling. (b) Waveforms that were used in the experiment. The thin almost sinusoidal pulse is the gate signal for pumping in, say, the "forward" direction, and the dashed $\pi$-shifted signal is for pumping in the "backward" direction. The low level of the gate pulse is zero. The thick lines are the current signals corresponding to the arrowed path in the previous contour plot. The used gate signals were not perfectly sinusoidal but rather there was a dead time in the three signals such that the minima of the $E_{\mathrm{J}}$ 's and the extrema of the gate charge would coincide with better certainty. The shallow dips in the current signals compensate for the inductive cross coupling. (c) Contour plot of the measured current at a constant voltage of $250 \mu \mathrm{V}$ against the relative phase differences between the signals with the pumping signal being applied at $2 \mathrm{MHz}$. The blue circle is the optimal choice for pumping "forward" while the red circle is the optimal point for pumping "backward." The amplitude was set large (over 400e) and the variation in current was $150 \mathrm{pA}$. This operation point is far from optimal, but we still obtain a clear modulation for calibration purposes. The color scales in (a) and (c) are relative and in units of pA.

not only the mutual inductances $M_{i j}$ between coil $i$ and SQUID $j$, which were $M_{11}=30 \mathrm{pH}, M_{12}=2 \mathrm{pH}, M_{21}=3 \mathrm{pH}$, and $M_{22}=50 \mathrm{pH}$, but also the proper offsets at any given time, i.e., the background fluxes threading the loops. This measurement does not fully demonstrate to which extent it is possible to suppress the Josephson energy.

In the beginning of an ideal pumping cycle the $E_{\mathrm{J}}$ 's of both loops are set as close to zero as possible and the position of the gate determines the ground state. We see that initially the ground state of the island is an eigenstate of 

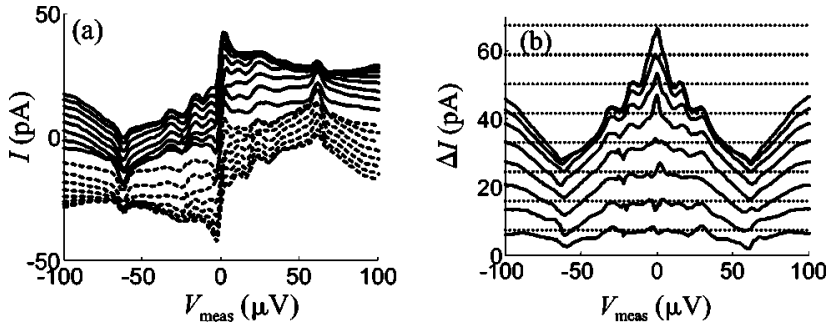

FIG. 3. (a) Examples of measured $I$ - $V$ curves with the pumping signal applied at $3 \mathrm{MHz}$. The gate charge (in $2 e$ units) varies between 4 and 34. The solid curves correspond to pumping forward and the dashed curves correspond to pumping backward. Here $V_{\text {meas }}$ is the measured value of voltage over the pump. (b) Difference of current, $\Delta I$, in the $I-V$ curves of (a) for pumping in opposite directions. The dotted lines indicate the expected values.

charge. We then adiabatically "open" one of the SQUIDs, i.e., move to the tip of the, say, horizontal arrow in Fig. 2(a) which means that the $E_{\mathrm{J}}$ of SQUID 1 is maximized while for the other it is still zero. We stay at the tip of the arrow for some time and start to either decrease or increase the gate charge $n_{\mathrm{g}}$ depending on the direction we have chosen. When the gate reaches its extremum we "close" the SQUID again. Now, if everything has been adiabatic, the system is still in its ground state. The charge is again a good quantum number at this point but since the position of the gate is different, the number of charges is different, too. The only possibility is that the excess charges have tunneled through the SQUID whose $E_{\mathrm{J}}$ has been nonvanishing during the cycle. The $E_{\mathrm{J}}$ of the second SQUID is then opened and the gate put back to its initial position. Finally, the second SQUID is also closed. The number of Cooper pairs pumped is given by the difference between the integers closest to the high and low levels of the gate charge. Fixing the low level and sweeping the high level should result in a $2 e$-periodic staircase in the pumped current.

The phase of the gate determines naturally the direction, i.e., a $180^{\circ}$ phase shift reverses the pumped current. Figure 2(c) illustrates the measured behavior of the current when the relative phases between the pulses are varied. The phase of coil 1 is fixed at $180^{\circ}$ and the phases of the other two are swept. The two circles shown are the optimal choices for pumping. Note that the extrema of current are indeed $180^{\circ}$ apart in the gate as expected and the optimal choices are the ones illustrated in Fig. 2. For practical reasons we were forced to use frequencies in the $\mathrm{MHz}$ range, but in the present pumping scheme it is possible to increase the value of current conveniently by increasing the gate amplitude. We tried out different shapes of pulses such as a mere sinusoidal gate signal, but it was found that it is better to keep the gate constant while the $E_{\mathrm{J}}$ is not maximized, which is in accordance with the adiabaticity requirement. In practice we have arranged for a $15 \%$ dead time between the flux pulses, although no systematic optimization of the pulses was performed.

Figure 3(a) shows an example of characteristic $I-V$ curves (i.e., current-voltage curves) with the pumping signal being applied at $f=3 \mathrm{MHz}$. The effect of the change of direction is shown. The curves correspond to eight different values of
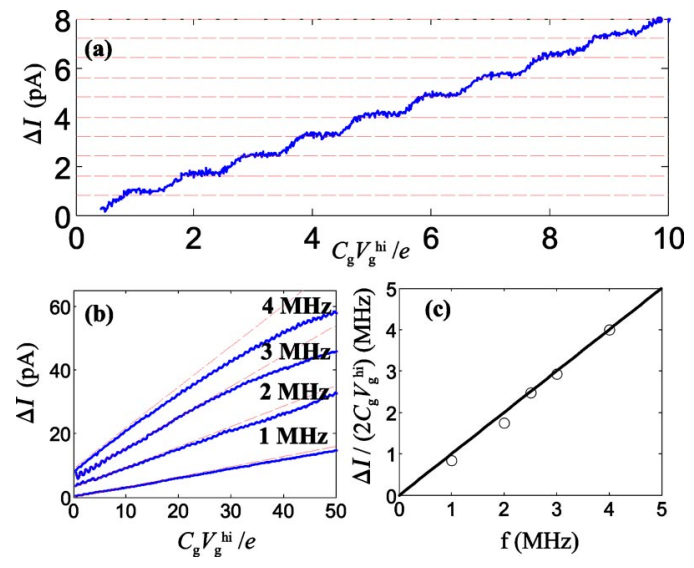

FIG. 4. (a) Difference $\Delta I$ in current of forward and backward pumping at $2.5 \mathrm{MHz}$ against the high level of the gate signal $V_{\mathrm{g}}^{\text {hi }}$ with the low level at zero. The dashed lines are drawn at 2 ef intervals. (b) Large gate amplitude behavior of $\Delta I$ at a few frequencies. The dashed lines show the expected gate dependence, i.e., their slope is $2 e f$. The curves are offset for clarity. (c) Fitted slopes to the data of the previous plots up to $V_{\mathrm{g}} C_{\mathrm{g}} / e=10$ are shown by circles. The solid line indicates the expected behavior. The voltage bias point was around $10 \mu \mathrm{V}$ in all the above plots.

gate amplitude. We see immediately that a leakage current exists on top of the pumped current that is on the same order or less than the pumped current. The $I-V$ curves, however, clearly shift and the curves for pumping in opposite directions are far apart. The total current flowing through the device is a sum of two contributions, one being the leakage supercurrent that can be associated with the dynamical phase of the wave function and the other being the less trivial pumping contribution attributable to the geometric phase. If one assumes that the leakage is the same for the pumping in both directions at a definite voltage bias point, then the difference between the $I-V$ curves should be twice the magnitude of current pumped in this case. Figure 3(b) reveals that at low voltages (tens of $\mu \mathrm{V}$ ) and at smaller amplitudes this pumping contribution is indeed close to the expected level shown with dotted lines. The leakage current, which is due to the nonideal environment and flux control, is undesirable from an application point of view, but the physical phenomenon is clearly visible. The voltage bias is not sufficiently good to eliminate the leakage, i.e., the $P(E)$ curve $^{8}$ for tunneling events is not sufficiently peaked at the origin.

Figure 4(a) shows the measured behavior of $\Delta I$ at 2.5 $\mathrm{MHz}$ versus the high level of gate voltage with the low level set to zero. The current may be seen to increase in clear steps. The expected height of a step is twice the pumped current, i.e., 4 ef which in this case is some $1.6 \mathrm{pA}$. Since we sweep the high level of the gate signal and not just the amplitude with constant offset, the steps should occur at $2 e$ intervals in the gate charge. However, due to random parity changes (quasiparticle "poisoning") at time scales that are much shorter than our measurement time scale $(0.1 \mathrm{~s})$ but longer than the pumping cycle $\left(10^{-6} \mathrm{~s}\right)$, we observe the time average of two $2 e$-periodic staircases that are shifted by $e$ in the gate charge. For instance, in Ref. 9 the tunneling time for quasiparticles was estimated to be $10 \mu \mathrm{s}$ in a similar struc- 
ture while in Ref. 10 it was some $10^{-2} \mathrm{~s}$ for a coupled system of two superconducting transistors with one grounded. We were unable to measure the corresponding time in our setup, but, based on this supporting evidence, we argue that the transport of current is due to Cooper pairs since the order in which the $E_{\mathrm{J}}$ 's are manipulated changes the direction of current. The quasiparticles effectively shift the gate charge by $e$ but rarely enough such that the pumping is undisturbed on the level of precision of the present measurement. If this interpretation is made, then one sees that the obtained results are in very good agreement with theory. Figure 4(b) illustrates the measured large amplitude behavior of the pumped current at frequencies between 1 and $4 \mathrm{MHz}$. We see that the current lacks behind the prediction with increasing frequency and amplitude. At $1 \mathrm{MHz}$ no clear bending of the curve is seen up to gate amplitude of $40 e$, while at $4 \mathrm{MHz}$ the performance starts to degrade after $10 e$. One can observe by looking at Fig. 3(b) that the "bending" is more pronounced at larger bias voltage values (voltage is on the order of $10 \mu \mathrm{V}$ in Fig. 4) while no visible bending happens up to amplitudes of $68 e$ when $V \approx 0$. Small amplitude behavior in Fig. 4, however, is linear aside from the steps with a slope of $2 e f$. Figure 4(c) shows the slopes obtained from linear fits to the data of Fig. 4(a) and the first ten steps of Fig. 4(b). One sees that the agreement is again good.

The above results prove that the flux and voltage driven pumping of Cooper pairs is experimentally possible in a single-island device. However, in order to serve as a practical device the leakage current needs to be taken care of as well as the quasiparticle poisoning. The quasiparticles may possibly be handled by either quasiparticle "traps" or by BCS gap profile engineering. ${ }^{9}$ As to the reduction of the leakage, several options exist. One option is the engineering of the electromagnetic environment such that the voltage biasing is good also at frequencies on the order of the charging energy. This would result in de $I-V$ characteristics heavily peaked at zero voltage with negligible leakage current. Another way to cut down the leakage is to fabricate a longer chain of junctions. A multiloop SQUID would possibly improve the suppression of $E_{\mathrm{J}}$ without increasing the number of controls. Improved rf engineering would also be of benefit in arranging the flux pulses. To conclude, the results are encouraging in spite of several nonidealities observed and the pumping of Cooper pairs with flux control looks much more attractive than with a mere multiple gate voltage control.

We thank H. Sipola and S. Franssila for help with the measurement setup and device fabrication, and A. Anthore, T. Heikkilä, P. Helistö, and M. Paalanen for useful discussions. The Academy of Finland and EU IST-FET-SQUBIT2 are acknowledged for financial support.
*Electronic address: antti.niskanen@vtt.fi

${ }^{1}$ L. J. Geerligs, S. M. Verbrugh, P. Hadley, J. E. Mooij, H. Pothier, P. Lafarge, C. Urbina, D. Esteve, and M. H. Devoret, Z. Phys. B: Condens. Matter 85, 349 (1991).

${ }^{2}$ M. W. Keller, J. M. Martinis, N. M. Zimmerman, and A. H. Steinbach, Appl. Phys. Lett. 69, 1804 (1996).

${ }^{3}$ D. Vion, A. Aassime, A. Cottet, P. Joyez, H. Pothier, C. Urbina, D. Esteve, and M. H. Devoret, Science 296, 886 (2002).

${ }^{4}$ Y. A. Pashkin, T. Yamamoto, O. Astafiev, Y. Nakamura, D. V. Averin, and J. S. Tsai, Nature (London) 421, 823 (2003).

${ }^{5}$ J. M. Shilton, V. I. Talyanskii, M. Pepper, D. A. Ritchie, J. E. F. Frost, C. J. B. Ford, C. G. Smith, and G. A. C. Jones, J. Phys.:
Condens. Matter 8, L531 (1996).

${ }^{6}$ A. O. Niskanen, J. P. Pekola, and H. Seppä, Phys. Rev. Lett. 91, 177003 (2003).

${ }^{7}$ Yu. Makhlin, G. Schön, and A. Shnirman, Rev. Mod. Phys. 73, 357 (2001).

${ }^{8}$ G.-L. Ingold and Yu. V. Nazarov, in Single Charge Tunneling, edited by H. Grabert and M. H. Devoret (Plenum Press, New York, 1992), pp. 21-106.

${ }^{9}$ J. Aumentado, M. W. Keller, J. M. Martinis, and M. H. Devoret, Phys. Rev. Lett. 92, 066802 (2004).

${ }^{10}$ J. Männik and J. E. Lukens, Phys. Rev. Lett. 92, 057004 (2004). 